\title{
Treatment Associated Interstitial Pulmonary Toxicity of Temozolomide Plus Bevacizumab for Locally Advanced Solitary Fibrous Tumor
}

\section{Heissner $\mathbf{K}^{1, \star}$, Horger $\mathbf{M}^{2}$, Spengler $\mathbf{W}^{1}$, Steger $\mathbf{V}^{3}$, Kanz $\mathrm{L}^{1}$, Biro $\mathrm{D}^{4}$ and Kopp HG}

${ }^{1}$ Department of Medical Oncology, Hematology, Immunology, Rheumatology and Pulmology, Medical Center II, South West German Comprehensive Cancer Center, University Hospital of Tuebingen, Germany

${ }^{2}$ Department of Radiology, Diagnostic and Interventional Radiology, University Hospital of Tuebingen, Germany

${ }^{3}$ Department of Thoracic and Cardiovascular Surgery, University Hospital of Tuebingen, Germany

${ }^{4}$ Department of Endocrinology, Diabetology, Angiology, Nephrology and Clinical Chemistry, University Hospital of Tuebingen, Germany

\begin{abstract}
Solitary Fibrous Tumors (SFT), previously termed haemangiopericytoma, are rare soft tissue sarcomas (STS). They usually grow slowly and show indolent behaviour in terms of malignancy. Surgery in localized disease is the only curative approach. While metastatic disease occurs rarely, locally advanced SFT often require systemic treatment. Systemic treatment for inoperable SFT is not standardized, and chemotherapy displays only modest activity. Temozolomide plus bevacizumab is a relatively active treatment option. Its advantages are prolonged tumor stabilisation combined with an overall low toxicity profile. We report the first case of treatment related interstitial pulmonary toxicity caused by temozolomide in combination with bevacizumab during treatment of SFT.
\end{abstract}

Keywords: Solitary fibrous tumor; Hemangiopericytoma; Toxicity; Pulmonary; Temozolomide; Bevacizumab; Chemotherapy

\section{Introduction}

A 74 year old man presented with a right pleural mass, which was recognized in 2013 on a plain chest x-ray (Figure 1) after emergency coronary catheter intervention because of severe three-vessel coronary artery disease. Histological examination of tumor tissue gained during bronchoscopy confirmed the diagnosis of a solitary fibrous tumor. During exploratory thoracotomy the tumor was found to be technically inoperable. Since November 2013 the tumor was growing slowly but continuously without intervention. During that time the patient lived without major restrictions. Since May 2015, the patient became symptomatic with progressive shortening of breath because of worsening restrictive lung function (Figure 2) and dramatic decrease of vital capacity (total lung capacity: 3,51 (normal: 71 ), vital Capacity: 1,4l (normal: 3,81)).

Because of already impaired left ventricular function, doxorubicin was contraindicated. We decided to treat the patient with a combination regimen using temozolomide plus bevacizumab as described in detail by Park et al. [1], consisting of 28 day cycles of $150 \mathrm{mg} / \mathrm{m}^{2}$ temozolomide on days 1-7, 15-21 and bevacizumab $5 \mathrm{mg} / \mathrm{kg}$ on days 8,22 .

7 cycles temozolomide (cumulative dose: $14.700 \mathrm{mg} / \mathrm{m}^{2}$ ) plus bevacizumab (cumulative dose: $70 \mathrm{mg} / \mathrm{kg}$ ) were administered with overall good tolerability and without relevant toxicity. Thereafter the patient developed progressive shortness of breath and an increasing CRP value up to $11 \mathrm{mg} / \mathrm{dl}$ (normal: $<0.5 \mathrm{mg} / \mathrm{dl}$ ). A CT scan of the thorax supported by densitometry showed bilateral increase of lung parenchyma density (Figure 3). Myocardial ischemia, pulmonary embolism, and congestive heart failure were ruled out. Treatment with prednisolone was initiated ( $1 \mathrm{mg} / \mathrm{kg}$ body weight). Shortness of breath improved while the patient still needed home oxygen therapy. A follow up CT scan 7days after corticoid treatment (Figure 4), already shows an decrease of lung parenchyma density.

\section{Discussion}

Solitary fibrous tumors represent approximately $2 \%$ of all STS [2]. SFT can develop in any anatomical site, including intracranial tissues. SFT usually grow slowly and patients remain asymptomatic for a prolonged period of time. Neither immunhistochemical nor histological nor radiological features are sufficient parameters for

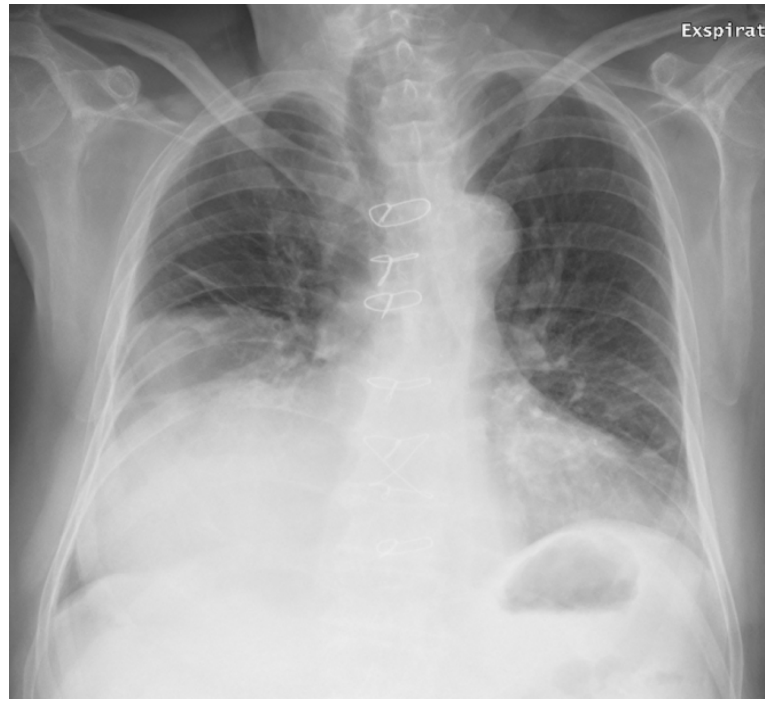

Figure 1: Plain chest X-ray at primary diagnosis September 2013: Flad shading of the right middle- and lower lobe. The finding is compatible with a solid mass or encapsulated pleural effusion after coronary angiography. Mediastinal shading suspect for a solid mass or retrosternal goiter.

prediction of the clinical behaviour. While metastatic disease is an exception, locally advanced and infiltrative growth is common.

Treatment of choice is the complete resection with a high chance of

${ }^{*}$ Corresponding author: Klaus Heissner, Center for Soft Tissue Sarcoma, GIST and Bone Tumors, Southwest German Tumor Center Comprehensive Cancer Center (CCC), Otfried-Müller-Str. 10, 72076 Tübingen, Germany, Tel: +49 70712982714 Fax: +49 707129 4399; E-mail: klaus.heissner@med.uni-tuebingen.de

Received December 17, 2015; Accepted January 13, 2016; Published January 18 2016

Citation: Heissner K, Horger M, Spengler W, Steger V, Kanz L, et al. (2016) Treatment Associated Interstitial Pulmonary Toxicity of Temozolomide Plus Bevacizumab for Locally Advanced Solitary Fibrous Tumor. J Pulm Respir Med 6: 314. doi:10.4172/2161-105X.1000314

Copyright: ( 2016 Heissner K, et al. This is an open-access article distributed under the terms of the Creative Commons Attribution License, which permits unrestricted use, distribution, and reproduction in any medium, provided the original author and source are credited. 
Citation: Heissner K, Horger M, Spengler W, Steger V, Kanz L, et al. (2016) Treatment Associated Interstitial Pulmonary Toxicity of Temozolomide Plus Bevacizumab for Locally Advanced Solitary Fibrous Tumor. J Pulm Respir Med 6: 314. doi:10.4172/2161-105X.1000314

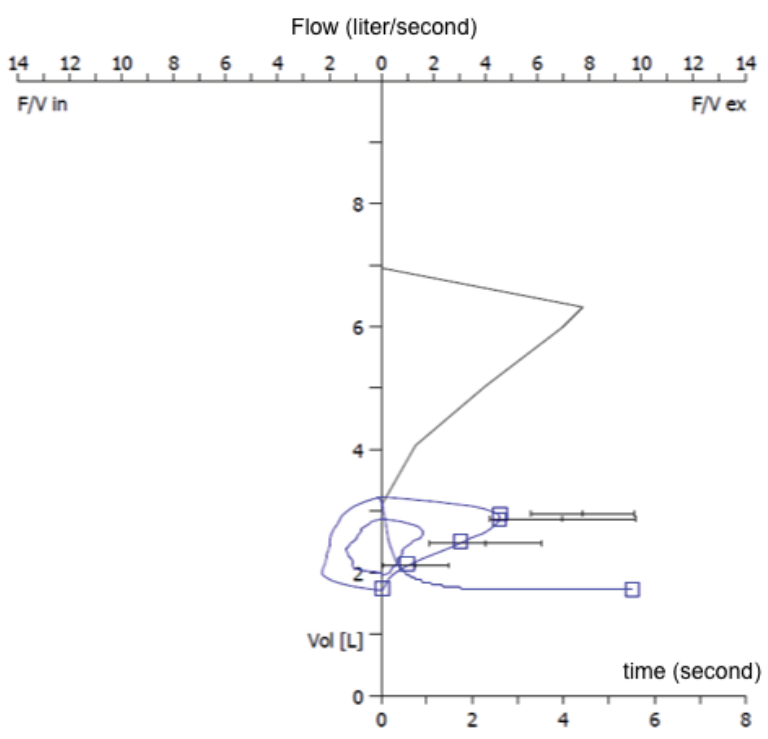

\begin{tabular}{|c|c|c|c|c|c|c|}
\hline & unit & normal & patient & $\begin{array}{c}\% \\
\text { patient/ } \\
\text { normal }\end{array}$ & patient & $\begin{array}{c}\% \\
\text { patient/ } \\
\text { normal } \\
11 / 2015\end{array}$ \\
\hline iVC & liter & 3.99 & 2.92 & 72.3 & 1.69 & 42,5 \\
\hline FVC & liter & 3,85 & 2,57 & 66 & 1.64 & 42.7 \\
\hline FEV1 & liter & 2.90 & 2,3 & 77.7 & 1.43 & 49.2 \\
\hline MEF 50 & liter/second & 4 & 3.18 & 78.3 & 2.3 & 57.6 \\
\hline RV & liter & 2.73 & 2.01 & 74.8 & 1.65 & 60.6 \\
\hline TLC & liter & 6.98 & 5.05 & 72.3 & 3.27 & 46.8 \\
\hline DLCOC & mmol/min/kPa & 8.71 & 8.02 & 92.2 & 3.52 & 41.0 \\
\hline
\end{tabular}

Figure 2: Bodyplethysmographie at primary diagnosis (2013) and after manifest pulmonary toxicity (2015) showing massiv worsening of pre-existing restrictive lung disease as such small vessel disease. Legend: iVC=inspiratory vital capacity; FVC=forced vital capacity; FEV1=one second capacity; MEF 50=mean expiratory flow; RV=residual volume; TLC=total lung capacity, DLCOc=diffusing capacity.

curability [3]. Local relapse seems to be associated, but not limited to, incomplete resection [4]. As slow growing tumors, SFT are difficult to treat with systemic treatment. If patients respond to treatment, disease stabilization is much more common compared to partial responses. Chemotherapeutic options are therefore reserved for palliative situations with locally advanced, inoperable and/or metastatic disease. Because of the rarity of SFT, there are no generally accepted recommendations for standard treatment. Small case series with a maximum of 30 patients represent the basis for treatment recommendations. Temozolomide plus bevacizumab is an attractive treatment for some patients. The regimen was published to be well tolerated and clinically beneficial with a median progression free survival (mPFS) of 9.7 months [1]. Other treatment approaches were reported to be equally or even less active. Some examples include anthracyclin-based regimens with an mPFS of 4 months [5], dacarbacin alone resulted in PFS of 2-11 months [6], pazopanib was recently reported to achieve mPFS of 4.7 months [7] and sunitinib a PFS of 6 months [8].

The herein presented patient was initially treated with temozolomide plus bevacizumab, achieving prolonged disease stabilisation at the cost of serious interstitial lung toxicity. At this point it is worthwhile to note, that temozolomide and bevacizumab are both used off-label in
SFT. Bevacizumab is used in a wide spectrum of indications [9]. The safety profile is well characterized and pulmonary toxicity is typically limited to haemorrhage. The probability that the use of bevacizumab in the presented case played a role for pulmonary toxicity is very low.

Temozolomide is approved for the treatment of newly diagnosed glioblastoma multiforme and refractory anaplastic astrocytoma [10]. It is an orally available DNA alkylating agent. Safety has been extensively studied for this drug. The far majority of adverse events is linked to hematotoxicity, nausea, and vomiting. FDA prescription reveals grade III or IV toxicity below 30\% [10,11]. Temozolomide triggered hepatotoxicity has been described in literature and therefore been recognized by physicians. In consequence, regular liver function testing is recommended [12]. Neyns reported no increased incidence of late toxicity in patients with brain tumors using temozolomide [13]. Meanwhile some data suggest survival benefit in brain tumor patients using long-term temozolomide treatment [14]. A patient reported by Spence and colleagues had accidental overdose of temozolomide [15]. Nausea, vomiting, diarrhoea and haematological adverse events occurred in this patient. No pulmonary (long-term) toxicity was observed. While nausea and vomiting where the most common complains respectively, respiratory disorders including lung 
Citation: Heissner K, Horger M, Spengler W, Steger V, Kanz L, et al. (2016) Treatment Associated Interstitial Pulmonary Toxicity of Temozolomide Plus Bevacizumab for Locally Advanced Solitary Fibrous Tumor. J Pulm Respir Med 6: 314. doi:10.4172/2161-105X.1000314
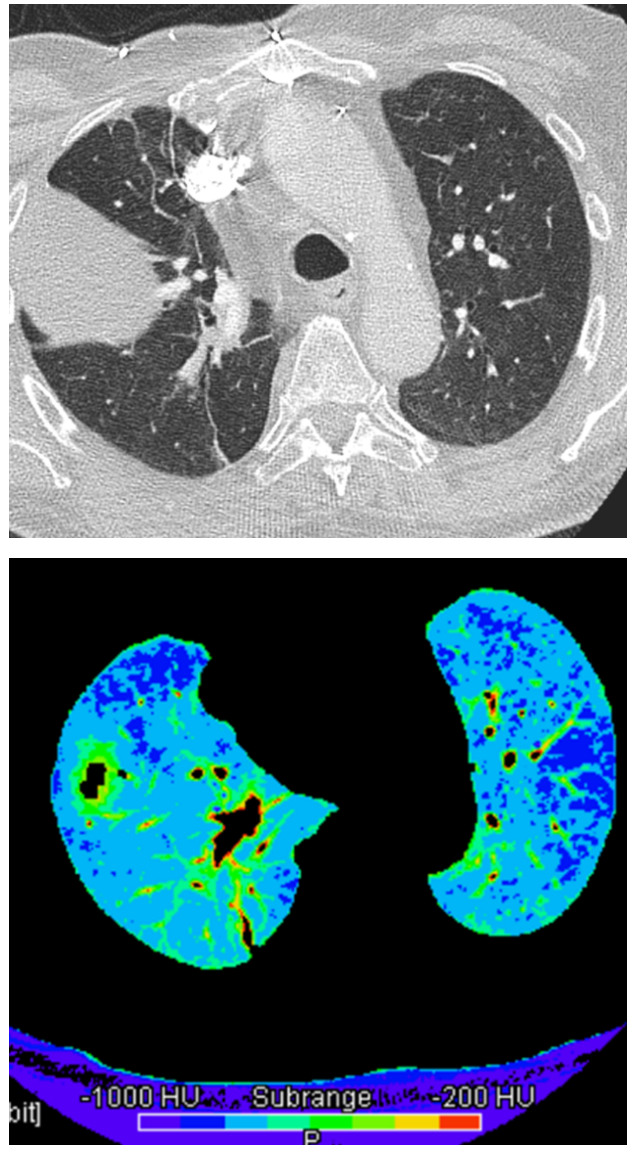

Figure 3: CT-scan thorax including lumen volumetric for parenchymal quantification (densitometry) November 2015 after 7 cycles temozolomide plus bevacizumab: intrathoracic tumor (sliced). Diffuse decrease in lung parenchyma density compatible to viral infection or medication toxicity.

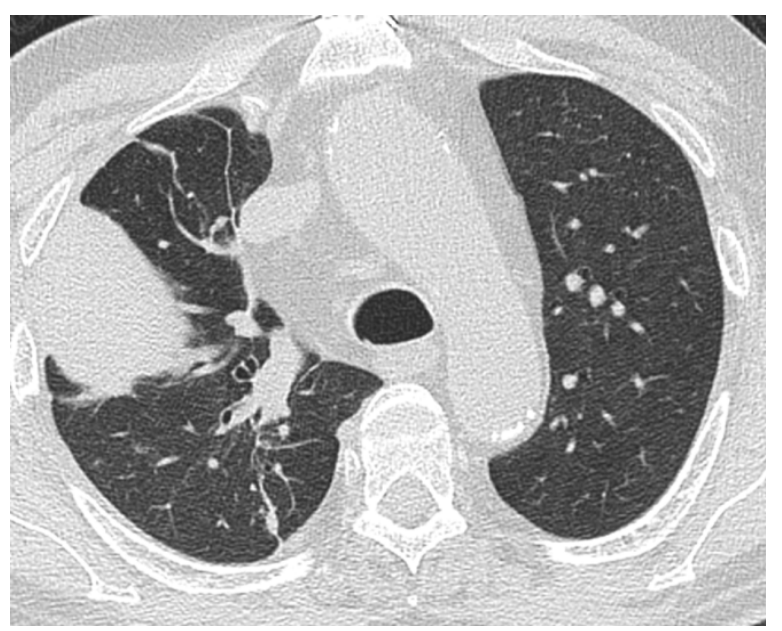

Figure 4: CT-scan thorax November 2015 after 7 days corticosteroid treatment: no change in tumor size (sliced) but already regredient parenchyma density compared to Figure 3 . infections, coughing and dyspnoea have been reported repetitively, but affect less than $5 \%$ of patients. Individual patients suffering from mild interstitial pneumonitis most often do not require dose reductions or stop of treatment [16]. Pneumocystis juvenii pneumonia prophylaxis is only recommended in newly diagnosed glioblastoma multiforme during temozolomide treatment parallel to brain radiation because of CD4 selective lymphocytopenia and concomitant treatment with corticosteroids [10]. Interestingly, considering the voluntarily reported adverse events using temozolomide in post market experiences, reports on pulmonary disorders like intestinal (hypersensitivity) pneumonitis, alveolitis and pulmonary fibrosis are well documented [10,17-22]. Clinical manifestation and CT findings with diffuse patchy ground glass opacities with intralobular septal thickening were similar among the reports and to our findings. Probably they are all different expressions of massive acute pulmonary parenchymal injury. Despite one case, all patients recovered rapidly after application of corticosteroids.

While antineoplastic therapy related pulmonary toxicity is a common problem in daily patient care, alkylating agents such as temozolomide have been rarely identified as potential cause. All cases of serious pulmonary toxicity published became evident in patients treated with brain tumors. Unfortunately, evidence based criteria for therapy related pulmonary diseases have not been published yet. Drug exposure, typical imaging findings and the exclusion of other causes led us to the conclusion of temozolomide associated toxicity. Rapid responsive to corticosteroid therapy supports the diagnosis ex juvantibus remediis.

We would like to sensitize physicians that SFT patients under temozolomide plus bevacizumab may develop serious pulmonary toxicity, even after prolonged period of exposure to both drugs. Shortness of breath, cough or dyspnoea in these patients must therefore be evaluated with pulmonary function testing and high resolution CT scans of the thorax.

\section{References}

1. Park MS, Patel SR, Ludwig JA, Trent JC, Conrad CA, et al. (2011) Activity of temozolomide and bevacizumab in the treatment of locally advanced, recurrent, and metastatic hemangiopericytoma and malignant solitary fibrous tumor. Cancer 117: 4939-4947.

2. Gold JS, Antonescu CR, Hajdu C, Ferrone CR, Hussain M, et al. (2002) Clinicopathologic correlates of solitary fibrous tumors. Cancer 94: 1057-1068.

3. Demicco EG, Park MS, Araujo DM, Fox PS, Bassett RL, et al. (2012) Solitary fibrous tumor: a clinicopathological study of 110 cases and proposed risk assessment model. Mod Pathol 25: 1298-1306.

4. Baldi GG, Stacchiotti S, Mauro V, Dei Tos AP, Gronchi A, et al. (2013) Solitary fibrous tumor of all sites: outcome of late recurrences in 14 patients. Clin Sarcoma Res 3: 4

5. Stacchiotti S, Libertini M, Negri T, Palassini E, Gronchi A, et al. (2013) Response to chemotherapy of solitary fibrous tumour: a retrospective study. Eur J Cancer 49: $2376-2383$

6. Stacchiotti S, Tortoreto M, Bozzi F, Tamborini E, Morosi C, et al (2013) Dacarbazine in solitary fibrous tumor: a case series analysis and preclinical evidence vis-a-vis temozolomide and antiangiogenics. Clin Cancer Res 19 5192-5201.

7. Maruzzo M, Martin-Liberal J, Messiou C, Miah A, Thway K et al. (2015) Pazopanib as first line treatment for solitary fibrous tumours: the Royal Marsden Hospital experience. Clin Sarcoma Res 5: 5.

8. Stacchiotti S, Negri T, Libertini M, Palassini E, Marrari A, et al. (2012) Sunitinib malate in solitary fibrous tumor (SFT). Ann Oncol 23: 3171-3179.

9. www.avastin.com

10. www.drugs.com/pro/temodar

11. Bae SH, Park MJ, Lee MM, Kim TM, Lee SH, et al. (2014) Toxicity profile of 
Citation: Heissner K, Horger M, Spengler W, Steger V, Kanz L, et al. (2016) Treatment Associated Interstitial Pulmonary Toxicity of Temozolomide Plus Bevacizumab for Locally Advanced Solitary Fibrous Tumor. J Pulm Respir Med 6: 314. doi:10.4172/2161-105X.1000314

temozolomide in the treatment of 300 malignant glioma patients in Korea. $\mathrm{J}$ Korean Med Sci 29: 980-984.

12. Sarganas G, Orzechowski HD, Klimpel A, Thomae M, Kauffmann W, et al. (2012) Severe sustained cholestatic hepatitis following temozolomide in a patient with glioblastoma multiforme: case study and review of data from the FDA adverse event reporting system. Neuro Oncol 14: 541-546.

13. Roldan Urgoiti GB, Singh AD, Easaw JC (2012) Extended adjuvan temozolomide for treatment of newly diagnosed glioblastoma multiforme. J Neurooncol 108: 173-177.

14. Neyns B, Chaskis C, Joosens E, Menten J, D'Hondt L, et al. (2008) A multicenter cohort study of dose-dense temozolomide (21 of 28 days) for the treatment of recurrent anaplastic astrocytoma or oligoastrocytoma. Cancer Invest 26: 269277

15. Spence AM, Kiem HP, Partap S, Schuetze S, Silber JR, et al. (2006) Complications of a temozolomide overdose: a case report. J Neurooncol 80 : $57-61$.

16. Abrey LE, Olson JD, Raizer JJ, Mack M, Rodavitch A, et al. (2001) A phase II trial of temozolomide for patients with recurrent or progressive brain metastases. $J$
Neurooncol 53: 259-265.

17. Koschel D, Handzhiev S, Leucht V, Holotiuk O, Fisseler-Eckhoff A, et al. (2009) Hypersensitivity pneumonitis associated with the use of temozolomide. Eur Respir J 33: 931-934.

18. Balzarini L, Mancini C, Marvisi M (2014) A fatal case of acute interstitial pneumonia (AIP) in a woman affected by glioblastoma. Curr Drug Saf 9: 73-76.

19. Kim TO, Oh IJ, Kang HW, Chi SY, Ban HJ, et al. (2012) TemozolomideAssociated Bronchiolitis Obliterans Organizing Pneumonia Successfully Treated with High-Dose Corticosteroid. J Korean Med Sci 27: 450-453.

20. Maldonado F, Limper AH, Lim KG, Aubrey MC (2007) Temozolomide-associated organizing pneumonitis. Mayo Clin Proc 82: 771-773.

21. Constantinidou A, Jones RL, Olmos D, Thway K, Fisher C, et al. (2012) Conventional anthracycline-based chemotherapy has limited efficacy in solitary fibrous tumour. Acta Oncol 51: 550-554.

22. Park MS, Ravi V, Conley A, Patel SR, Trent JC, et al (2013) The role of chemotherapy in advanced solitary fibrous tumors: a retrospective analysis. Clin Sarcoma Res 3: 7 